\title{
KIR gene polymorphism study in the Uygur population in Xinjiang, China
}

\author{
G.-Y. Lin and Y.-B. Wang \\ No. 474 Hospital of Chinese PLA, Urumqi, China \\ Corresponding author: G.-Y. Lin \\ E-mail: lgy474@yeah.net \\ Genet. Mol. Res. 13 (1): 1813-1822 (2014) \\ Received December 18, 2012 \\ Accepted August 28, 2013 \\ Published March 17, 2014 \\ DOI http://dx.doi.org/10.4238/2014.March.17.9
}

\begin{abstract}
We analyzed killer cell immunoglobulin-like receptor $(K I R)$ gene polymorphisms and genotype and haplotype characteristics in the Uygur population, a non-nomadic ethnic group found in Xinjiang, China, to provide a basis for studies on relationships between KIRs and diseases in this group. Sequence-specific primer PCR was used to detect the KIR gene in 84 Uygur individuals. A standard genotype and haplotype analysis was conducted using Hsu's standards. Sixteen KIR genes were detected; the 3DL3, 2DL4, 3DL2 genes were found in all individuals. The 2DL1, 3DP1, 2DP1, 2DL3 genes were relatively common, followed by 2DS4, 3DL1, 2DL5, 2DS2, 2DL2, 2DS1, 2DS5, and 3DS1. The 2DS3 gene had the lowest frequency. A total of 19 genotypes were detected, the $\mathrm{AJ}(2,2), \mathrm{AH}(5,2)$ and $\mathrm{M}(2,8)$ genotypes were relatively common, with frequencies of 25, 10.7 and $10.7 \%$, respectively; they were followed by $\mathrm{P}(2,17)$, AI $(1,5), \mathrm{H}(2,4)$, and $\mathrm{C}(5,3)$, with frequencies of $9.52,5.95,5.95$, and $4.76 \%$, respectively. The U $(17,21)$, and $\mathrm{T}(8,8)$ genotypes, found in four individuals, had not been reported in the Han Chinese population. Eleven haplotypes were detected; the most common haplotype $2(\mathrm{~N}=74)$ was accounting for $48.7 \%$, followed by haplotype $5(\mathrm{~N}=24)$, accounting for $15.8 \%$. In addition, three new genotypes were found, for which haplotype analysis could not be performed based on existing standards. We conclude
\end{abstract}


that the Xinjiang Uygur population has unique KIR gene frequency, genotype frequency and haplotype frequency distributions; there also appears to be new genotypes and haplotypes in this population.

Key words: Killer cell immunoglobulin-like receptor; Uygur; Gene polymorphism; Polymerase chain reaction sequence-specific primers

\section{INTRODUCTION}

Killer cell immunoglobulin-like receptor (KIR) is a group of specifically recognized human major histocompatibility antigen molecule receptor expressed on the surface of NK cells and $\mathrm{T}$ cells. It has been shown to be a highly diverse immune regulation gene family after the discovery of the HLA, and it has important immunomodulatory effects on NK cells and $\mathrm{T}$ cells. The KIR encoding gene is located on chromosome 19q13.4, and the family group includes 18 genes, namely KIR1D, KIR2DL1-5, KIR2DS1-5, KIR3DL1-3, KIR3DS1, Xv, X and KIR2DP1. Xv, X, and KIR2DP1 are pseudogenes, and KIR2DL4, KIR3DL2 and KIR3DL3 are framework genes (Zhang et al., 2003a). Studies have found that the KIR genes have genetic polymorphisms and that there is a rich diverse KIR receptor library in different individuals and NK cell cloning expression. The expression pattern of NK cells directly regulates the ability to clear foreign and harmful substances, and the individual KIR genotype is the most critical factor to determine its expression, which plays an important role in the body's natural immunity and acquired immunity. Therefore, KIR genotyping helps us to study its function in certain diseases (Zhang et al., 2003b). Previous studies (Khakoo and Carrington, 2006) have shown that the KIR gene polymorphism is associated with certain infectious diseases, autoimmune diseases, cancer susceptibility, and pregnancy. The regulatory effects in hematopoietic stem cell transplantation have been of great concern in the clinical field. We worked in the multi-ethnic region of Xinjiang to determine the distribution characteristics of the KIR gene polymorphism in the Xinjiang Uyghur population, where we used the sequence-specific primer PCR method (PCR-SSP) to perform the KIR genotyping and genotype and haplotype analysis of 84 cases of Xinjiang Uygur volunteers. This study will lay the foundation for further studies of the association between the KIR gene polymorphism and certain diseases in different groups of people.

\section{MATERIAL AND METHODS}

\section{Design}

Our study was a prospective analysis. DNA was analyzed in vitro using molecular biology detection methods. Unrelated individuals participated in this randomized trial. Our study was carried out from November 2009 to September 2010 in the kidney transplant tissue typing room of the blood purification center in the People's Liberation Army No. 474 Hospital.

The kidney transplant HLA matching donors and recipients gave their written informed consent. A 4-mL sample of venous whole blood was collected from donors and recipients and kept in EDTA anticoagulant. Our room stored the genomic DNA from 84 independent Uygur individuals from the Xinjiang region, and the volunteer samples were randomly selected. DNA extraction was in strict accordance with the instructions of the PROTRANS 
DNA rapid extraction kit, which included a total of 17 steps to complete. The $\mathrm{A}_{260} / \mathrm{A}_{280}$ ratio of the DNA in the samples was from 1.8 to 2.0 . The genomic DNA samples were stored at $-20^{\circ} \mathrm{C}$ for later analysis.

\section{Reagents and instruments}

The KIR PCR-SSP kit was provided by Tianjin Sau Peng Biotechnology Development Co., Ltd., China. The internal reference control was the conservative fragments of the human growth hormone gene, and the amplification product of the internal reference was 588 bp. Taq DNA polymerase was provided by Promega Corporation (Beijing China). The DNA extraction kit was the German PROTRANS DNA extraction kit provided by Beijing San Taihua Biotechnology Company, China. The PCR amplification instrument was the Bio-Rad type of UK. The electrophoresis instruments such as the DDY-BC and WD-9413B gel imaging analyzers were supplied by the Beijing Liuyi Instrument Company, China.

\section{Detection and genotyping of $K I R$ genes}

The PCR-SSP genotyping method for KIR genes was adopted in all samples collected. KIR2DL1-KIR2DL5, KIR2DS1-KIR2DS5, KIR3DL1-KIR3DL3, KIR3DS1, and the two pseudogenes KIR2DP1 (aka KIRZ) and KIR3DP1 (divided into KIRX, and KIRXv) were detected in the KIR gene loci. The PCR-SSP amplification were designed based on the KIR gene sequence and genotyping literature design, and a total of 32 pairs were synthesized by Tianjin Sau Peng Biotechnology Co., Ltd.. The amplifications were performed in the Bio-Rad PCR amplification instrument. The PCR system volume was $10 \mu \mathrm{L}$ per well, and there were 16 wells for one person. The system contained $160 \mu \mathrm{L}$ dNTP buffer, $20 \mu \mathrm{L}$ genomic DNA, $1.2 \mu \mathrm{L}$ Taq DNA polymerase $(5 \mathrm{U} / \mu \mathrm{L})$. The reaction conditions were as follows: $96^{\circ} \mathrm{C}$ for $2 \mathrm{~min}, 96^{\circ} \mathrm{C}$ for $20 \mathrm{~s}$ and $68^{\circ} \mathrm{C}$ for $60 \mathrm{~s}$, for a total of 5 cycles; $96^{\circ} \mathrm{C}$ for $20 \mathrm{~s}, 64^{\circ} \mathrm{C}$ for $50 \mathrm{~s}$ and $72^{\circ} \mathrm{C}$ for $45 \mathrm{~s}$, for a total of 10 cycles; and $96^{\circ} \mathrm{C}$ for $20 \mathrm{~s}, 61^{\circ} \mathrm{C}$ for $50 \mathrm{~s}$ and $72^{\circ} \mathrm{C}$ for $45 \mathrm{~s}$, for a total of 18 cycles. Final extension was at $72^{\circ} \mathrm{C}$ for $5 \mathrm{~min}$. The PCR products were loaded on a $2.5 \%$ agarose gel. The DYY-BC-type electrophoresis apparatus was used for electrophoresis at $160 \mathrm{~V}$ for $12 \mathrm{~min}$. The WD-9413B gel imaging analyzer (UV) was used for the gel image analysis.

\section{Genotype and haplotype analysis}

We adopted the 36 KIR genotypes A to AJ, which were first defined in Caucasians by Hsu et al. (2002) and the designated 23 common haplotypes using standard typing methods. The KIR haplotype combinations were projected according to the genotypes. When the same genotype had 2 haplotype combinations, it was calculated according to the common haplotype literature (Hsu et al., 2002). In the 23 common haplotypes in the literature, haplotype 1 and haplotype 2 were type A haplotype, and the other 21 haplotypes were type B haplotype. The comprehensive classification criteria were that all the haplotypes contained 3DL3, 2DL4 and 3DL2, one haplotype contained 2DL2 or 2DL3. One haplotype contained 3DP1 or 3DP1v. The haplotype B carried one or more of the genes KIR2DL5, KIR2DS1, KIR2DS2, KIR2DS3, KIR2DS5, and KIR3DS1, but the haplotype A did not include these genes.

Main outcome measurement: 16 KIR gene loci including KIR2DL1-KIR2DL5, KIR2DS1-KIR2DS5, KIR3DL1-KIR3DL3, KIR3DS1, and the two pseudogenes KIR2DP1 (aka 
KIRZ) and KIR3DP1 (divided into KIRX, and KIRXv) were detected. The KIR gene frequencies, genotype frequencies and haplotype frequencies were calculated by the allele-counting method.

\section{Statistical analysis}

According to the literature (Hsu et al., 2002), the KIR gene frequency (f) was measured by counting: $\mathrm{pf}(\%)=$ the positive gene number/the total number in the research group. The KIR gene frequency was calculated as follows: $g f=1-(1-f)^{1 / 2}$, genotype frequency was the genotype positive number $n_{1} / \mathrm{N}$, the haplotype frequency was the single haplotype positive number $\mathrm{n}_{2} / 2 \mathrm{~N}$, and $\mathrm{N}$ was the number of study individuals.

\section{RESULTS}

\section{KIR gene frequency distribution}

A total of 84 specimens were included in the result analysis. 3DL3, 2DL4 and 3DL2 genes existed in all individuals of the Xinjiang Uygur population, where the gene frequency was 1. 2DL1, 3DP1, 2DP1, and 2DL3 genes were common with gene frequency of 0.8457 , $0.8909,0.7328$, and 0.6915 , respectively. 2DS4, 3DL1, 2DL5, 2DS2, 2DL2, and 2DS1 genes were less common with frequencies of $0.6066,0.5635,0.3925,0.3274,0.3099$, and 0.2929 , respectively. The 2DS3 gene frequency was the lowest at 0.1619 (Table 1).

\section{KIR genotype frequencies}

There were 19 genotypes detected in the Xinjiang Uygur population. The AJ $(2,2), \mathrm{AH}$ $(5,2)$ and $\mathrm{M}(2,8)$ genotypes were more common with respective frequencies of $25.00,10.71$ and $10.71 \%$. They were followed by P $(2,17)$, AI $(1,5), \mathrm{H}(2,4)$, and C $(5,3)$ with the frequencies of $9.52,5.95,5.95$ and $4.76 \%$, respectively. In addition, there were three genotypes (NW1, NW2 and NW3) in 8 patients who could not be classified according to the related literature, and thus their haplotypes could not be determined. The frequencies were $3.57,4.7$ and $1.19 \%$, suggesting that new genotypes and haplotypes may exist. In contrast, the $U(17,21)$ and $T(8,8)$ genotypes in 4 patients had not been reported in the domestic literature of the Han population, and they possibly belonged to the Uighur population-specific genotypes with a frequency of $2.38 \%$ (Table 2).

\section{KIR gene haplotype frequencies}

The haplotype analysis could not be carried out in 8 (9.5\%) of 84 cases of the Uighurs in accordance with the method of Hsu et al. (2002), and 11 haplotypes were detected in the remaining 76 cases. They were haplotypes $1,2,3,4,5,6,8,9,14,17$, and 21 . The most common haplotype $2(\mathrm{~N}=74)$ was found in $54.4 \%(74 / 152)$, and it was followed by haplotype 5 $(\mathrm{N}=24)$, which accounted for $17.6 \%(24 / 152)$ and haplotype $8(\mathrm{~N}=14)$ accounting for $9.2 \%$ $(14 / 152)$. Haplotypes 7, 10, 11, 12, 13, 15, 16, 18, 19, 20, 22, and 23 were not detected in this study. Eighty-two cases were haplotype A with a frequency of $53.9 \%$, and the ratio of the haplotype A and B was 1.17:1. The haplotypes could not be calculated with three genotypes according to the method of Hsu et al. (2002), suggesting that there may be new haplotypes (Table 3).

$K I R$ gene frequencies in the Xinjiang Uygur population compared with other populations are shown in Table 4. 


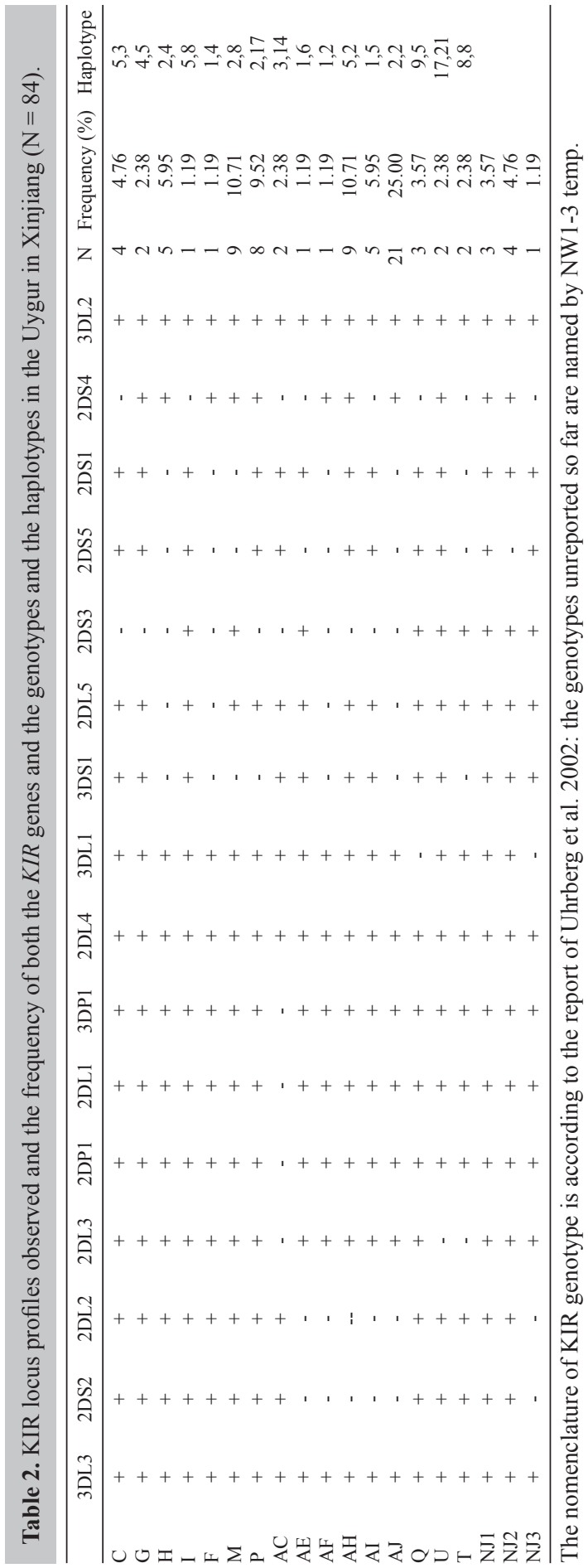




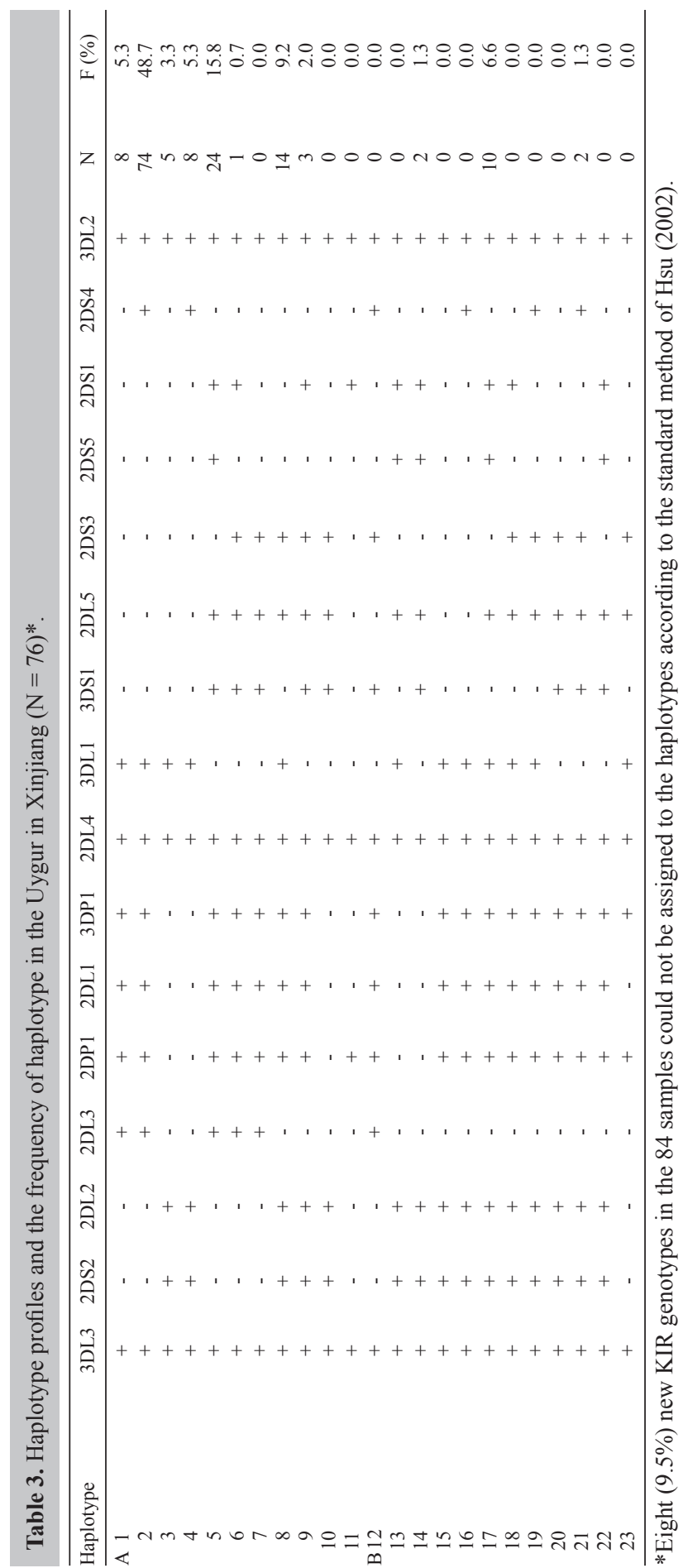




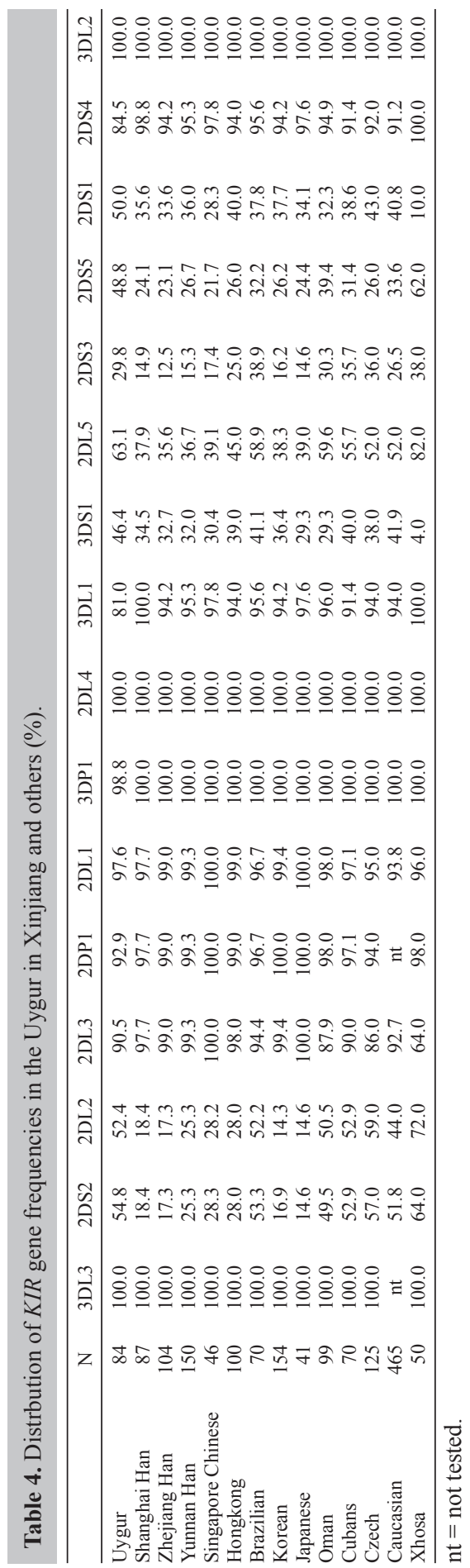




\section{DISCUSSION}

KIR genes have genetic polymorphisms. KIR genes are located on human chromosome $19 \mathrm{q} 13.4$, whose span is about $150 \mathrm{~kb}$. They have the head-to-tail arrangement and gather in clusters, their polymorphisms are encoded by the multi-gene family clusters, with diverse structure and function. The KIR loci contain multiple alleles, which can be expressed as the different $K I R$ genes and numbers in different ethnic groups and different areas. Different individuals have different numbers and types of KIR genes, so different individuals show different haplotypes. At present, many laboratories are dedicated to KIR genotyping and haplotype composition studies, and the KIR distribution has been reported in more than 140 groups of the world's population (Hsu et al., 2002; Rajalingam et al., 2002; Uhrberg et al., 2002; Jiang et al., 2005; Velickovic et al., 2006; Middleton et al., 2008; Pavlova et al., 2008).

Our study used the PCR-SSP method to detect the KIR gene loci in 84 cases of Xinjiang Uygur individuals who were the recipients and donors of a kidney transplant. We compared the test results with the other populations reported in the literature and obtained the KIR gene distribution characteristics of Xinjiang Uyghur population. 1) The detection rates of 3DL3, 2DL4, 3DL2 frame genes were 100\% in each group, the rates of 2DL3, 2DP1 2DL1, 3DP1 showed a higher gene frequency of $>90 \%$, and the results were consistent with the data in the rest of the world populations. 2) 2DS2, 2DL2, 2DL5, 2DS3, 2DS5 and 2DS1 showed lower frequencies, but they were significantly higher than those in the Han, South Korean and Japan populations and other Asian populations. The 2DS1 rate was significantly lower than that in the Xhosa people of South Africa. The 2DS2 and 2DL2 rates were similar with Oman, Caucasian and Cuban populations, but were lower than that in the Czech population. 3) The 3DS1 gene frequency of $46.4 \%$ was higher than in all comparable populations. 4) The 2DS3 gene frequency was $29.8 \%$, which was higher than in the Han, Japanese, South Korean, and Caucasian populations, but it was significantly lower than in the Brazilian, Czech Republic and South African Xhosa populations. 5) The 2DS5 gene frequency was $48.8 \%$ and significantly higher than the Han, South Korean, Singapore, and Japanese populations and the white population of United States, but it was significantly lower than in the South African Xhosa population. There were 19 KIR genotypes detected in the Xinjiang Uygur population, and it was lower than 30 genotypes in the Yunnan Han population (Su et al., 2008) and 26 genotypes in the Zhejiang Han population (Zhu et al., 2005), but higher than 18 genotypes in the Shanghai Han population (Zhang et al., 2003b). Among 19 kinds of KIR genotypes, the AJ $(2,2)$ genotype was the most common genotype, the AF $(1,2)$, I $(5,8), \mathrm{F}(1,4)$, and $\mathrm{AE}(1,6)$ genotypes were just behind. It was different with the AJ and AF dominating the Zhejiang, Shanghai, Jiangsu, and Yunnan populations (Zhu et al., 2005; Su et al., 2008; He et al., 2009), while the most common genotype in the Caucasian population was the AG $(1,1)$ genotype. In addition, we found 19 genotypes in 84 cases of Uyghur individuals, three genotypes (total eight cases) had not been reported and could not be named in accordance with the relevant literature (tentatively named NW1-3). In this study, in accordance with the method of Hsu et al. (2002), 11 haplotypes were found in the Uyghur population, with the most common haplotype 2 accounting for $48.7 \%$, followed by haplotypes 5 and 8 , accounting for 15.8 and $9.2 \%$, respectively. The haplotype A frequency was $53.9 \%$, and the A to B haplotype ratio was 1.17:1. According to reports, the most common haplotype in the Yunnan (Su et al., 2008) and Shanghai (Zhang et al., 2003b) Han populations was haplotype 2, but haplotype 1 was the most common in Caucasians. This was different from the finding of haplotype $\mathrm{A}$ in a higher 
proportion of the Yunnan, Zhejiang, Shanghai, Japan, South Korea populations. This was also different from the B haplotype domination in Australia's indigenous population, and it was more like the relatively balanced distribution in the white populations. This study found that three genotypes could not fit the haplotype combinations according to the standards of Hsu et al. (2002), suggesting that there may be new haplotype combinations. With regard to haplotype combinations, Zhang et al. (2003b) thought that some special genotypes may have more than one haplotype combination; for example, genotype A may have a combination of 1 and 10 or 3 and 7. Genotype $\mathrm{C}$ can either be a combination of 5 and 3 or a combination of 3 and 7. Genotype $\mathrm{C}$ may have a 5 and 3 or 1 and 14 combination. In this situation, we should give priority to more common haplotype combinations in this population and finally confirm it by expanding the pedigree or DNA profiles.

Our study suggested that different races may have different $K I R$ genes and genotype distributions. KIR gene distribution showed uniqueness in the Xinjiang Uygur population. There may be new KIR genotypes and haplotypes. The characteristics in the Uygur population were different from those of Asian populations such as Han and the African populations; they were more like the central Asian population characterized as Caucasians. Therefore, the KIR gene polymorphism analysis in the Xinjiang Uygur population reflects the phylogenetic relationship between races. This phenomenon of gene distribution differences provides the basic information for further studies of KIR genes in human genetics, transplantation immunology and genetic diseases.

\section{REFERENCES}

He CT, Li L and Zhang JQ (2009). Jiangsu Han blood donors killer cell immunoglobulin-like receptor gene diversity research. Clin. Lab. Sci. 27: 208-211.

Hsu KC, Liu XR, Selvakumar A, Mickelson E, et al. (2002). Killer Ig-like receptor haplotype analysis by gene content: evidence for genomic diversity with a minimum of six basic framework haplotypes, each with multiple subsets. $J$. Immunol. 169: 5118-5129.

Jiang K, Zhu FM, Lv QF and Yan LX (2005). Distribution of killer cell immunoglobulin-like receptor genes in the Chinese Han population. Tissue Antigens 65: 556-563.

Khakoo SI and Carrington M (2006). KIR and disease: a model system or system of models? Immunol. Rev. 214: 186-201.

Middleton D, Meenagh A, Moscoso J and Arnaiz-Villena A (2008). Killer immunoglobulin receptor gene and allele frequencies in Caucasoid, Oriental and Black populations from different continents. Tissue Antigens 71: 105-113.

Pavlova Y, Kolesar L, Striz I, Jabor A, et al. (2008). Distribution of KIR genes in the Czech population. Int. J. Immunogenet. 35: 57-61.

Rajalingam R, Krausa P, Shilling HG, Stein JB, et al. (2002). Distinctive KIR and HLA diversity in a panel of north Indian Hindus. Immunogenetics 53: 1009-1019.

Su PC, Yang TG and Zou H (2008). Polymorphism of killer cell immunoglobulin-like receptors gene in Yunnan Han population, China. Chin. J. Blood Transfus. 21: 268-272.

Uhrberg M, Parham P and Wernet P (2002). Definition of gene content for nine common group B haplotypes of the Caucasoid population: KIR haplotypes contain between seven and eleven KIR genes. Immunogenetics 54: 221-229.

Velickovic M, Velickovic Z and Dunckley H (2006). Diversity of killer cell immunoglobulin-like receptor genes in Pacific Islands populations. Immunogenetics 58: 523-532.

Zhang L, Hsu KC and Liu XR (2003a). Human KIR gene PCR-SSP genotyping and pedigree. Shanghai J. Immunol. 23: 99-103.

Zhang L, Hsu KC, Liu XR, Yang JQ, et al. (2003b). Killer Ig-like receptor gene content diversity and haplotype analysis in Chinese Han population in Shanghai. Zhonghua Yi Xue Yi Chuan Xue Za Zhi 20: 396-399.

Zhu FM, Jiang K, Lu QF, Zhang W, et al. (2005). Polymorphism of killer cell immunoglobulin-like receptors gene family in Zhejiang Han population. Zhongguo Shi Yan Xue Ye. Xue Za Zhi 13: 1109-1112. 